This document is the accepted manuscript version of the following article:

Bergamaschi, A., Andrä, M., Barten, R., Borca, C., Brückner, M., Chiriotti, S., ... Zhang, J. (2018). The MÖNCH detector for soft X-ray, high-resolution, and energy resolved applications. Synchrotron Radiation News, 31(6), 11-15. https://doi.org/10.1080/08940886.2018.1528428

\title{
The MÖNCH detector for soft X-ray, high resolution and energy resolved applications
}

A. Bergamaschi ${ }^{1}$, M. Andrä ${ }^{1}$, R. Barten ${ }^{1}$, C. Borca ${ }^{1}$, M. Brückner ${ }^{1}$, S. Chiriotti ${ }^{1}$, R. Dinapoli ${ }^{1}$, E. Fröjdh $^{1}$, D. Greiffenberg ${ }^{1}$, T. Huthwelker ${ }^{1}$, A. Kleibert ${ }^{1}$, M. Langer ${ }^{1}$, M. Lebugle ${ }^{1}$, C. Lopez-Cuenca ${ }^{1}$, D. Mezza ${ }^{1}$, A. Mozzanica ${ }^{1}$, J. Raabe ${ }^{1}$, S. Redford ${ }^{1}$, Ch. Ruder ${ }^{1}$, V. Scagnoli ${ }^{1,2}$, B. Schmitt ${ }^{1}$, X. Shi $^{1}$, U. Staub $^{1}$, D. Thattil ${ }^{1}$, G. Tinti ${ }^{1}$, C. F. Vaz ${ }^{1}$, S. Vetter ${ }^{1}$, J. Vila-Comamala ${ }^{1}$, J. Zhang ${ }^{1}$

\author{
${ }^{1}$ Paul Scherrer Institut, CH-5232 Villigen PSI, Switzerland \\ ${ }^{2}$ Laboratory for Mesoscopic Systems, Department of Materials, ETH Zurich, Switzerland
}

\section{Introduction}

The Swiss Light Source (SLS) Detector Group at the Paul Scherrer Institut (PSI) has successfully been developing hybrid detectors for synchrotron and X-ray Free Electron Laser (XFEL) applications for more than a decade [1]. Single photon counting detectors pushed significantly the development of hard $\mathrm{X}$-ray diffraction techniques, while charge integrating detectors promise to contribute in the next few years to science at XFELs and to extend their range of operation to synchrotron applications [2].

MÖNCH stands for Micropixel with enhanced pOsition rEsolution usiNg CHarge integration and takes its name from a Swiss mountain, like all other detectors developed at PSI. Its development started in 2013 in order to explore the limits of hybrid detectors in terms of electronic noise, dynamic range and spatial resolution [3].

Its unique $25 \mu \mathrm{m}$ pixel pitch allows direct conversion imaging with high spatial resolution that can be improved beyond the pixel pitch using interpolation techniques. Even at this small pitch, a bump-bond yield higher than $99.95 \%$ has been achieved on the $400 \times 400$ pixels $\left(1 \times 1 \mathrm{~cm}^{2}\right)$ MÖNCH0.3 prototype using the standard indium solder bump technique developed at PSI [4].

The outstanding low noise of less than $150 \mathrm{eV}$ r.m.s. allows the detection of $12 \mathrm{keV}$ photons with a Signal-to-Noise Ratio (SNR) higher than 80, $700 \mathrm{eV}$ photons with a SNR close to 5 or equivalently provides an energy resolution of about $350 \mathrm{eV}$ FWHM [5].

A large dynamic range up to $1 \mathrm{E} 3 \cdot 12 \mathrm{keV}$ photons or $1.5 \mathrm{E} 4 \cdot 700 \mathrm{eV}$ photons can be achieved by adjusting the gain of the detector such that the noise is always limited by the Poisson fluctuations on the number of photons rather than by the electronics.

Dynamic gain switching will be implemented in the next prototype in order to perform high intensity single shot experiments [6]. At the same time, the low leakage current given by the small pixels allows dead-time free operation at synchrotrons with an exposure time up to $5 \mathrm{~ms}$ even at room temperature, while for time resolved studies a frame rate of up to $3 \mathrm{kfps}$ can be achieved (6 kfps with a redesign of the readout board). Therefore, MÖNCH is suitable for both synchrotron and XFEL experiments.

Over the last few years three detector prototypes have been characterized and used for several pilot experiments at the SLS. In this work we present the preliminary results obtained in applications where we foresee a stronger impact for MÖNCH: soft X-ray detection, high resolution and energy resolved imaging at higher energies.

\section{Soft X-ray applications}

Many synchrotron applications rely on soft X-rays because of their high cross section for thin samples and for specimens containing light elements. Moreover many elemental edges which can be exploited for resonant scattering and spectromicroscopy are placed at energies below $2 \mathrm{keV}$, e.g. K-edges of light 
elements and L-edges of 3d transition metals which are relevant to study copper based superconductors or magnetic thin films $[7,8,9,10]$.

Single photon counting detectors are not suitable for low energies, since the charge produced by single photons (less than 200 electrons for $700 \mathrm{eV}$ photons in silicon) is comparable to the electronic noise and therefore cannot be discriminated. The low noise performance of MÖNCH allows the detection of $700 \mathrm{eV}$ X-rays with a SNR close to 5, which is normally defined as the single photon resolution limit [11]. Moreover, being a charge integrating detector, it can also detect multiple photons of energies lower than $700 \mathrm{eV}$, losing single photon resolution but producing images which scale linearly with the number of photons.

As an example of possible applications, figure 1 shows the resonant magnetic diffraction pattern of an array of ferromagnetic nanoislands arranged in a square lattice (also known as "artificial spin ice") [12] acquired at the RESOXS experimental station at the SLS [13]. The large dynamic range of the detector allows the simultaneous detection of first order Bragg peaks as well as magnetic peaks, the specular reflection and part of the direct beam. The detector can operate in absence of a fast shutter and even in case of saturation it does not suffer from radiation damage. The image also shows a wave pattern in the low intensity background which is due to a non-uniform entrance window of the detector (backplane doping rings). The backplane of the detector consists of a doped junction necessary to apply the bias voltage to the sensor which is insensitive to radiation and reduces the quantum efficiency at low energies. In collaboration with sensor manufacturers, we are trying to reduce the thickness of this entrance window down to at least $200 \mathrm{~nm}$ in order to achieve a quantum efficiency higher than $50 \%$ down to $500 \mathrm{eV}$ (compared to the current 10\% with an entrance window approximately $1 \mu \mathrm{m}$ thick).

The fast framing capability of the detector up to a few kfps and the dead-time free operation make fast scanning applications possible. Figure 2.a) shows one of the diffraction patterns acquired with MÖNCH at the SIM beamline of the SLS during a ptychographic scan of a magnetic FeGd microstructure on a SiN membrane using circularly polarized light [14].

The position sensitivity of MÖNCH allows performing ptychography experiments with a resolution which is not limited by the beam size as in STXM experiments. Figure 2.b shows the ptychographic reconstruction of the same magnetic FeGd microstructure using circularly polarized light, where areas of different intensities correspond to oppositely magnetized out-of-plane domains. For this reconstruction, 100 images with $1 \mathrm{~ms}$ exposure time have been summed up for each scanning step in order to improve the statistics and reduce the data throughput.

The position sensitivity also proved very useful for aligning the microscope and fast framing at $1 \mathrm{kHz}$ allowed the detection of vibrations of about $20 \mathrm{~nm}$ in the chamber which limit the ultimate achievable spatial resolution of the setup. 


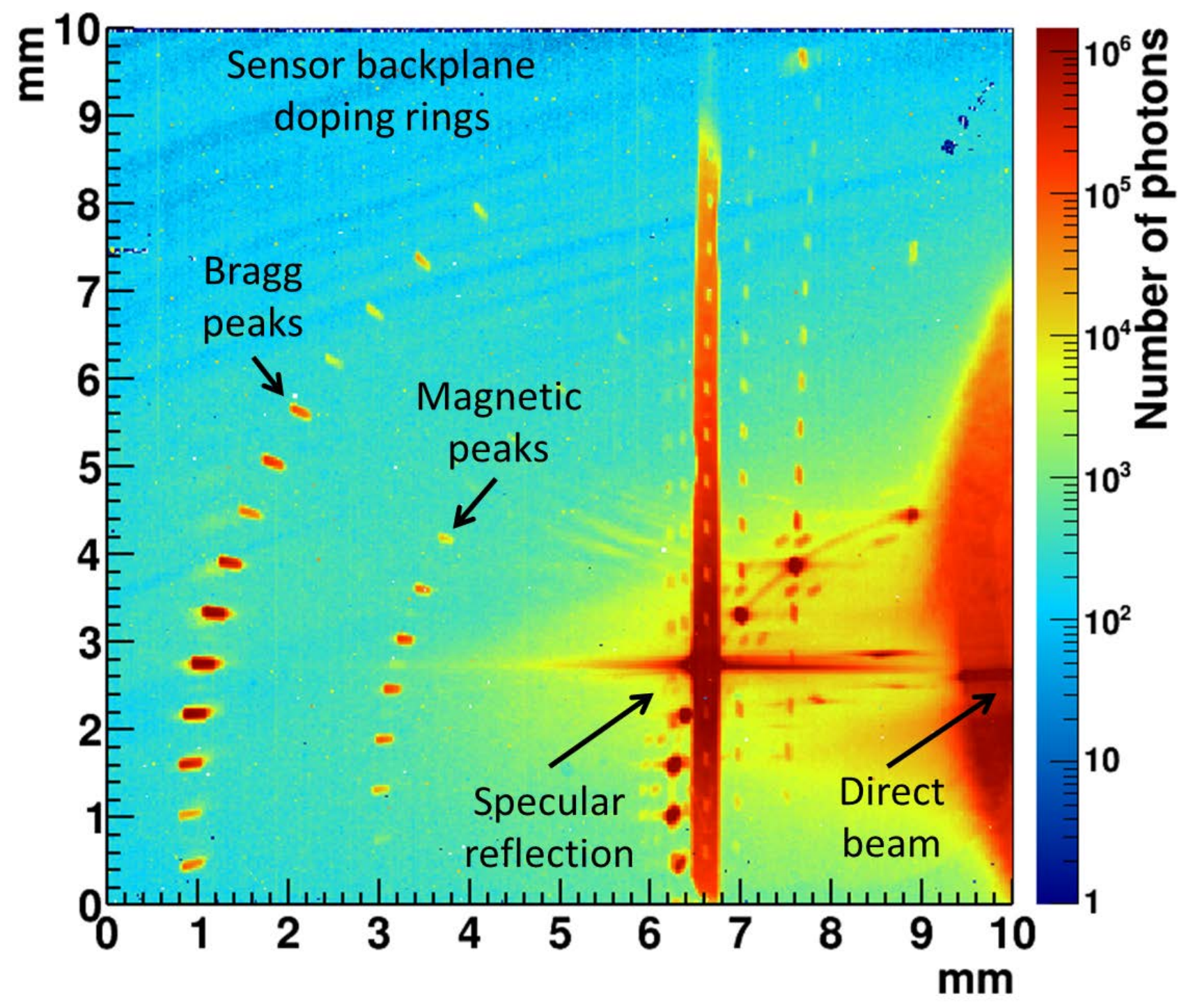

Figure 1: Image of the diffraction pattern of an artificial spin ice obtained at $708 \mathrm{eV}$. Thanks to the large dynamic range the Bragg and magnetic peaks can be detected simultaneously as well as the specular reflection and part of the direct beam. 


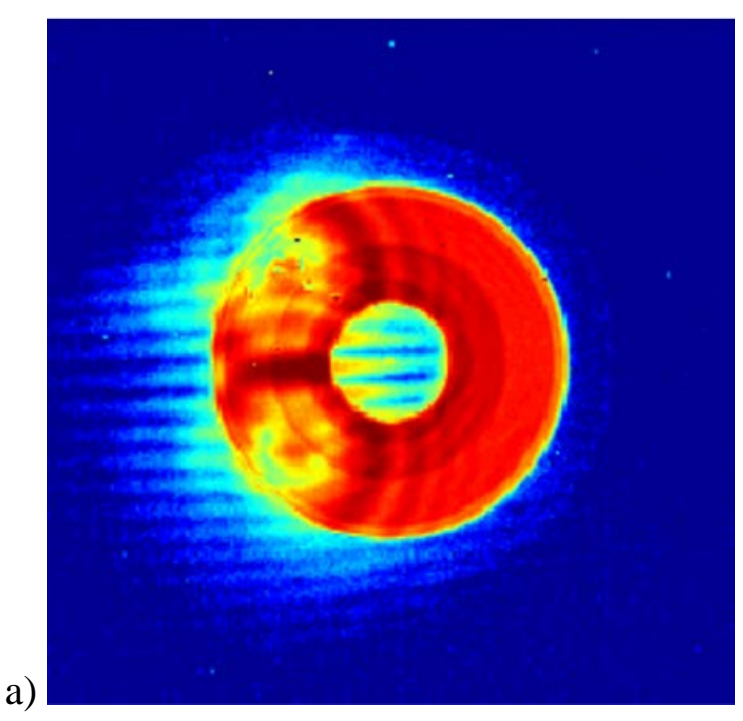

b)

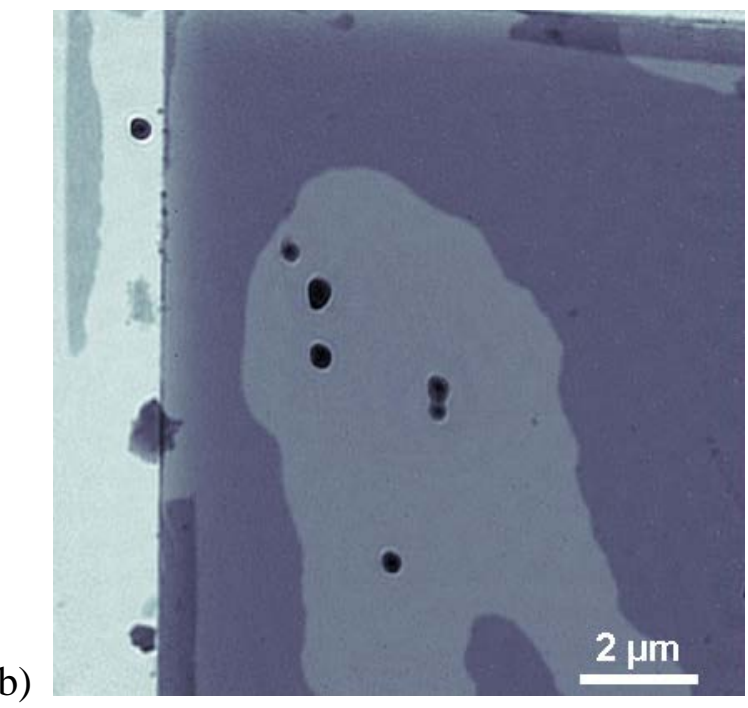

Figure 2: a) Image of the diffraction pattern of a magnetic FeGd nanostructure on a SiN membrane in a ptychographic scan acquired at $711 \mathrm{eV}$ using circularly polarized light. b) Ptychographic reconstruction of the same sample using 400 points arranged on a Fermat spiral.

\section{High resolution imaging}

Direct conversion detectors like MÖNCH provide a high Detective Quantum Efficiency (DQE) thanks to their single photon resolution. Moreover, the Point Spread Function (PSF), which is a figure of merit of the spatial resolution, is close to a single pixel i.e. $25 \mu \mathrm{m}$ in the case of MÖNCH. This allows low dose imaging with an adequate segmentation for many in situ or in vivo biological imaging applications as shown in [15].

The diffusion of the charge generated by the X-rays during the charge collection process inside the silicon sensor is of the order of $20 \mu \mathrm{m}$ for $320 \mu \mathrm{m}$ thick silicon sensors with 120V bias voltage for hard X-rays. When the cloud overlaps with two or more pixels, it is split among them (charge sharing) and in case of sparse photon hits it is possible to achieve a spatial resolution of a few microns using dedicated interpolation algorithms which exploit the analog information provided by the charge integrating readout of $\mathrm{MÖNCH} \mathrm{[16].} \mathrm{This} \mathrm{high} \mathrm{spatial}$ resolution cannot be obtained by reducing the pixel size due to technological limitations in the optical lithography involved in the sensor fabrication, the bump bonding process, the design of the readout electronics for hybrid pixel detectors and to the charge diffusion process itself.

Figure 3 shows the absorption image of a gold on silicon sample fabricated at PSI depicting the Eiger, Mönch and Jungfrau mountains acquired using $15 \mathrm{keV}$ photons at the Material Science beamline of the SLS [17]. The top image is acquired using the physical $25 \mu \mathrm{m}$ pixel size of the detector, while the bottom image is binned to $1 \mu \mathrm{m}$ virtual pixels after applying interpolation algorithms. The spatial resolution is clearly improved and the $7 \mu \mathrm{m}$ wide cross in the flag over the central peak is made visible.

The ultimate spatial resolution which can be obtained by applying interpolation techniques is mostly determined by the charge collection time and the SNR of single photons and therefore depends on several experimental parameters. The charge collection time mainly depends on the sensor thickness, the bias voltage and the X-ray energy. The energy also affects the SNR and 
consequently the accuracy on the measurement of charge sharing.

The spatial resolution is not uniform within the physical pixel: higher resolution is achieved closer to the edges, while it degrades in the center, due to the reduced charge sharing [15]. Usually the spatial resolution ranges between 1-4 $\mu \mathrm{m}$ r.m.s., depending on the position within the pixel and on the experimental parameters. This high spatial resolution allows single shot grating interferometry experiments by directly resolving the $4.7 \mu \mathrm{m}$ pitch fringes without the need of scanning an absorption grating [16].

The main limitation of interpolation is the low flux and fast framing required in order to detect isolated photons. With a maximum frame rate of $3 \mathrm{kfps}$, photons can be isolated in a $3 \times 3$ pixel cluster up to a maximum flux of about $5 E 5$ photons $/ \mathrm{s} / \mathrm{mm}^{2}$. An improvement in the maximum photon flux supported by interpolation can only come from a faster frame rate, which can be obtained by increasing the data transfer rate and by further parallelizing the data.

The acquisition at this low flux remains very dose effective, but requires long exposure times and does not allow the fast or time resolved experiments often performed at synchrotron beamlines. However the supported flux is comparable to the one provided e.g. by transmission microscopes where optics can be very inefficient, by fluorescence emission spectrometers or by microfocus X-ray tubes.

Interpolation has been demonstrated down to at least $2 \mathrm{keV}$, where the SNR of single photons is still sufficient to detect the charge sharing [4]. Interpolation in the tender X-ray energy range is exploited in a Von Hamos spectrometer installed at the PHOENIX beamline of the SLS for operation between 2 and $4 \mathrm{keV}$ [18]. The MÖNCH detector sits in the energy dispersive plane of the spectrometer, and directly records the spectra of the fluorescence lines. Figure 4.a shows the image of the chlorine $\mathrm{K}_{\alpha 1}-\mathrm{K}_{\alpha 2}$ doublet taken with the MÖNCH detector. The y axis is the energy dispersive direction $(99 \mu \mathrm{m} / \mathrm{eV})$, while the width of the recorded line in the $\mathrm{x}$ direction reflects the size of the segments of the segmented $\mathrm{Si}<111>$ crystal which is used in the spectrometer.

By interpolating to half of the pixel size (i.e. $12.5 \mu \mathrm{m}$ ), MÖNCH provides an energy resolution of about 125 meV per pixel, which could be pushed down of at least a factor two by binning to smaller virtual pixels. Figure 4.b shows the spectrum obtained by integrating the image in the horizontal direction, with an intrinsic energy resolution of the spectrometer of the order of 500 meV, i.e. still about 4 times larger compared to the one provided by the interpolated pixel size of the detector. The needs of this application match the performance of MÖNCH particularly well, i.e. low energy detection and high spatial resolution. Moreover the low flux due to the

fluorescence emission yield and optics efficiency can be well supported by interpolation without any attenuation.

\section{Energy resolved imaging}

When detecting isolated photons, on top of improving the position resolution, it is also possible to measure their energy with a resolution given by the electronic noise and by the cluster size i.e. about $700 \mathrm{eV}$ FWHM for $2 \times 2$ pixel clusters.

The spectroscopic performance combined with the position sensitivity can be exploited in a wide range of X-ray applications using polychromatic beams e.g. Laue diffraction, or color imaging (K-edge subtraction, material discrimination).

This capability is particularly interesting using X-ray tubes, where the flux is usually low enough to detect isolated photons.

Figure 5.a shows the image of a gold on silicon sample fabricated at PSI superimposed to silicon 
microspheres of a few hundred microns size acquired at a tungsten anode microfocus X-ray tube with $40 \mathrm{kVp}$ and $200 \mu \mathrm{A}$ current. The pitch of the Siemens star ranges between 60 and $0.5 \mu \mathrm{m}$ and interpolation is necessary to resolve the higher resolution region. Spokes down to about 10 $\mu \mathrm{m}$ pitch can be resolved. Artefacts are present in the image, partially due to the challenges given by the differences in charge sharing at different energies. Nevertheless, on top of improving the spatial resolution, it is also possible to produce a full energy spectrum for every pixel. Figure 5.b compares the measured X-ray spectra of regions with and without gold details. The $\mathrm{W}$ characteristics lines are visible in both cases, while it is possible to observe the Au Labsorption edges around $14 \mathrm{keV}$ for the gold on silicon regions.
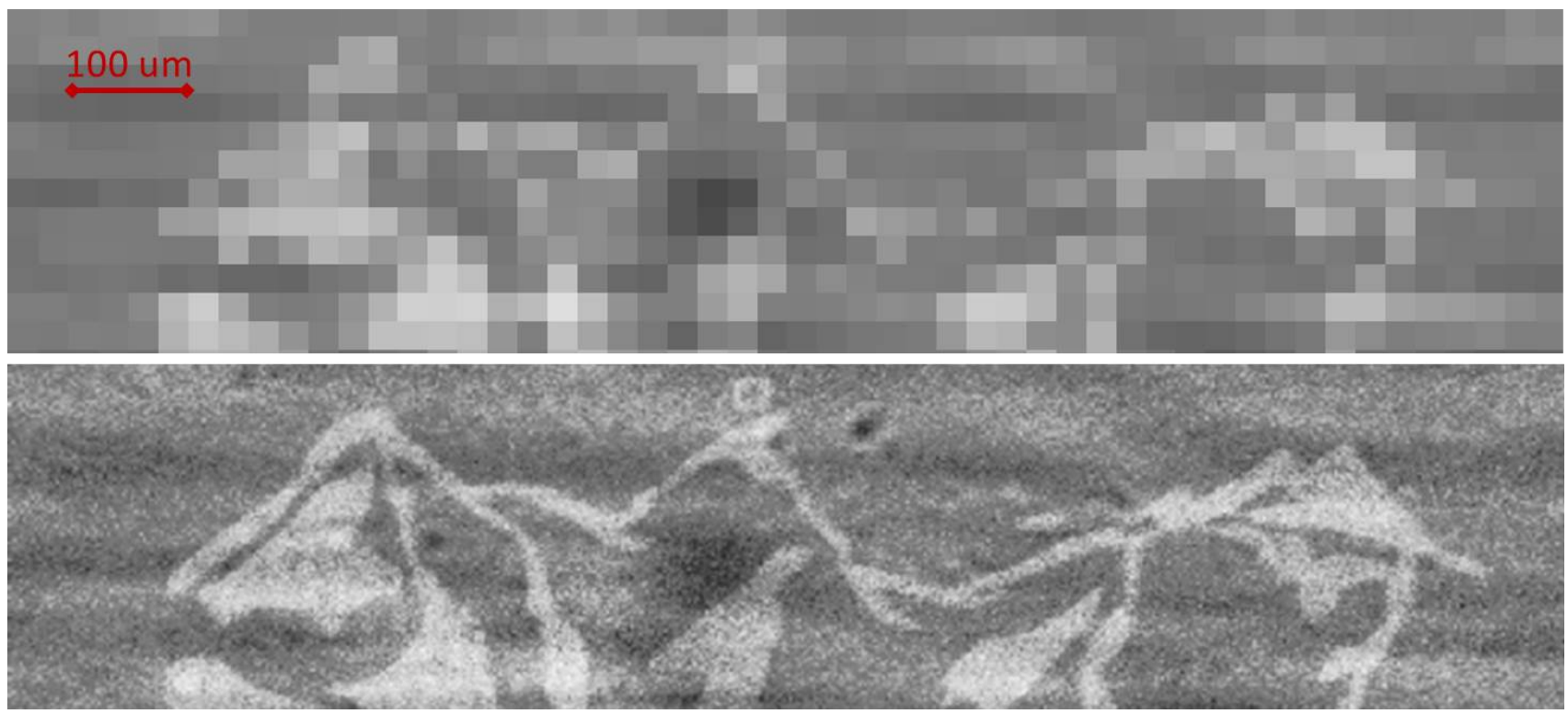

Figure 3: X-ray image of a gold on silicon sample fabricated at PSI depicting the Eiger, Mönch and Jungfrau mountains before (top) and after (bottom) interpolation to $1 \mu \mathrm{m}$ bins acquired with MÖNCH at $15 \mathrm{keV}$ at the Material Science beamline at the Swiss Light Source. The size of the Swiss flag on top of the central mountain is $25 \mu \mathrm{m}$ and the line width of the cross inside is only $7 \mu \mathrm{m}$ i.e. much smaller than the physical pixel size. 


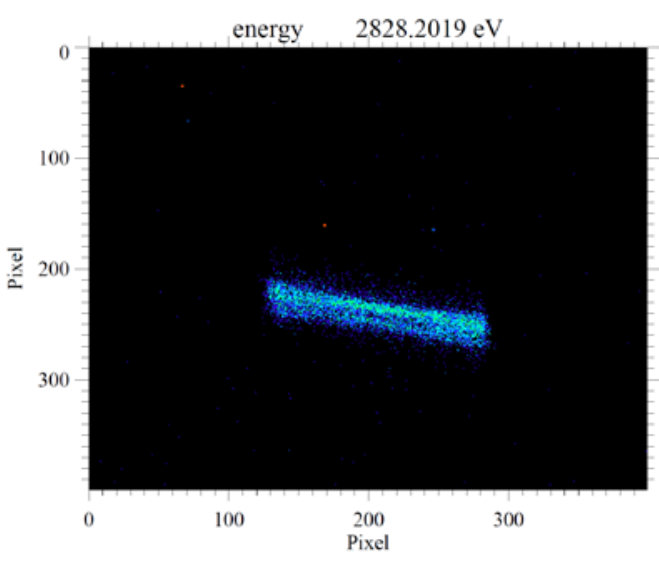

a)

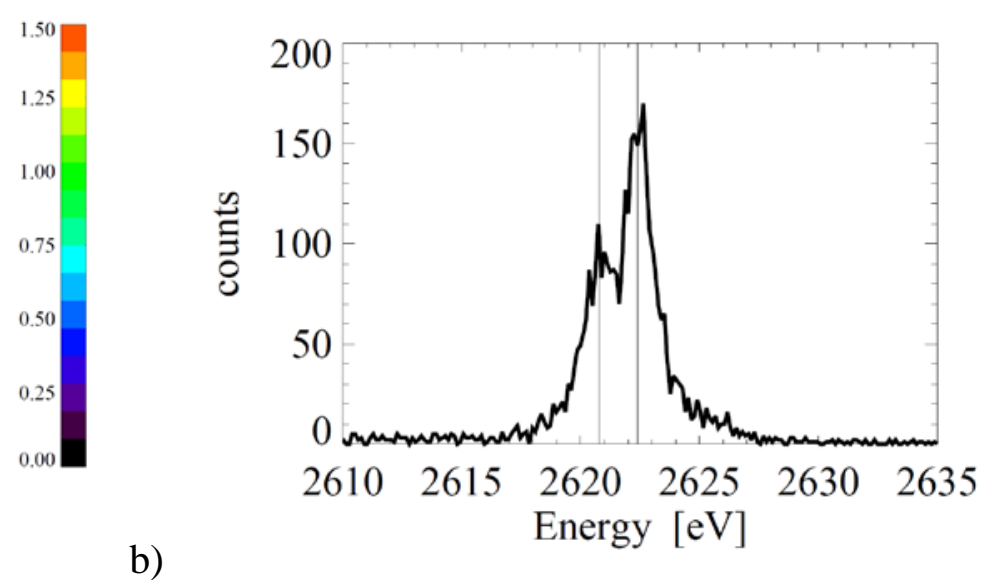

b)

Figure 4: Fluorescence lines of the chlorine $K_{\alpha 1-} K_{\alpha 2}$ doublet, as measured with the von Hamos spectrometer at an excitation energy of $2828.2 \mathrm{eV}$. a) Image of fluorescence lines taken with the MÖNCH detector. Data are presented in color scale as $\log _{10}($ counts +0.01$)$. The $y$-axis is the energy dispersive plane. One pixel $(12.5 \mu \mathrm{m})$ corresponds to $99.04 \mathrm{eV}$. b) Resulting spectrum of $K_{\alpha 1}-K_{\alpha 2}$ doublet. To derive the emission spectra from the MÖNCH raw data a region of 30 pixels of the $x$-axis has been averaged. The lines mark the theoretical energies of the $K_{\alpha 1}(2622.39 \mathrm{eV})$ and $K_{\alpha 2}(2620.78$ $e V)$ emission lines.

a)

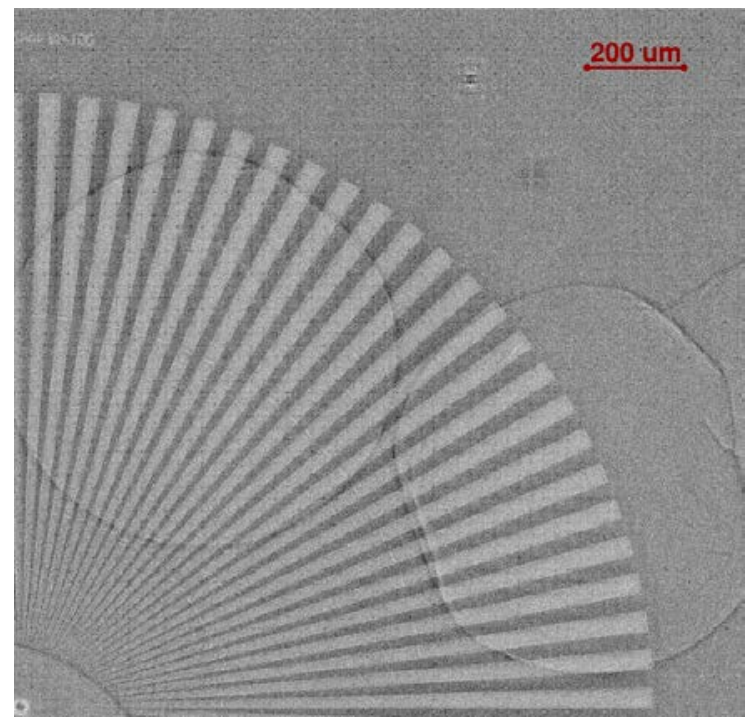

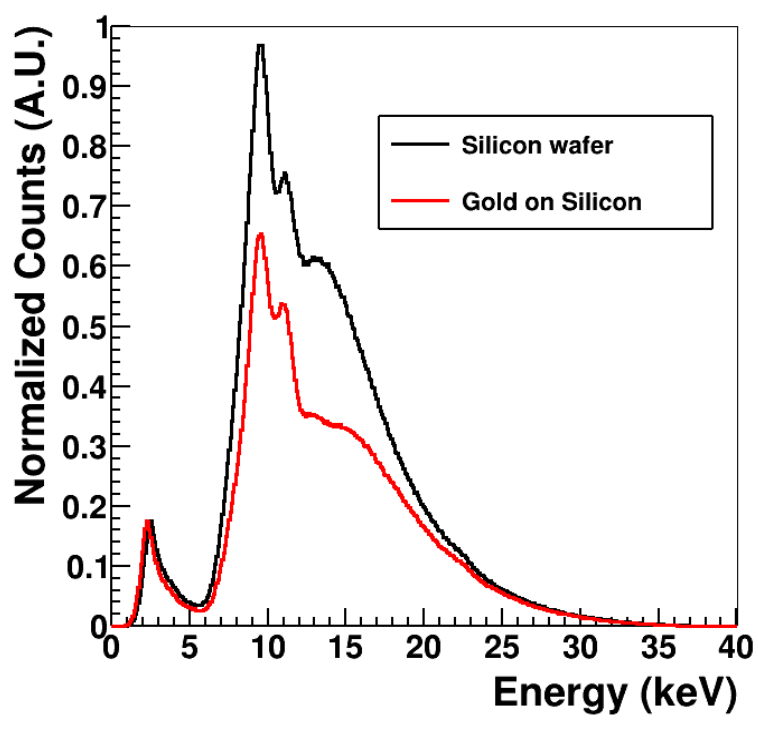

Figure 5: a) Interpolated image of a gold on silicon Siemens start with spokes ranging between 60 and $0.5 \mu \mathrm{m}$ pitch and silicon microspheres acquired at a microfocus $X$-ray tube and $b$ ) $X$-ray spectrum measured in regions with and without gold details. 


\section{Conclusions and perspectives}

The preliminary results obtained using MÖNCH have shown that two of the main flaws of hybrid detectors, i.e. high noise and poor spatial resolution, can be overcome.

The low noise of MÖNCH allows detection of soft X-rays with single photon resolution even below $1 \mathrm{keV}$. The main advantages of MÖNCH compared to monolithic detectors are the usability in a wide energy range, the robust and reliable operation common to all hybrid detectors, the fast framing and the large dynamic range. The detector does not suffer from radiation damage at low energies and dynamic gain switching will be implemented in the next MÖNCH prototype, allowing also for operation at XFELs.

We aim at further reducing the electronic noise in order to obtain single photon resolution down to about $500 \mathrm{eV}$. Some effort will also be required for improving the mechanics of the detector for in vacuum operation and for the optimization of the quantum efficiency of the sensors below $1 \mathrm{keV}$.

Regarding high resolution imaging, MÖNCH combines the high DQE given by direct conversion with high spatial resolution down to the micron level, which would be impossible to achieve without interpolation. The ultimate spatial resolution could further be improved by using silicon sensors up to $1 \mathrm{~mm}$ thickness, which also increase the quantum efficiency for hard $\mathrm{X}$-rays. Interpolation can satisfy the requirements of low flux applications, but faster frame rates would be desirable to increase the maximum flux. Applications requiring combined energy and spatial resolution will also be explored.

In the next years, the goal is to develop a large area detector targeting in particular soft X-ray applications for the ATHOS beamline of SwissFEL. We plan detector modules of almost 2 Mpixels built by butting two $2 \times 3 \mathrm{~cm}^{2}$ readout chips bump bonded to a single sensor.

Great challenges in the electronic design arise from the constraints in the pixel area, in the power consumption introduced by large number of pixels and in the parallelization required for fast readout.

Even with the relatively low $100 \mathrm{~Hz}$ operation at SwissFEL, the data throughput will be about $200 \mathrm{MB} / \mathrm{s}$ per module. For synchrotron operation, frame rates 5-10 times higher are desirable, implying a big effort in terms of processing, data reduction and storage.

It has been shown that the low noise and small pixel pitch of the MÖNCH detector open new possibilities for various X-ray applications ranging from soft X-ray detection to high resolution imaging. After successful experiments using prototypes, MÖNCH detectors of almost 2 Mpixels will be built, expecting that they will be used in a wide range of applications both at synchrotrons and XFELs.

\section{Acknowledgement}

For the soft X-ray ptychography experiment, we thank E. Kirk for the sample fabrication as well as M. Guizar-Sicairos and the cSAXS group for the support with reconstruction algorithms for the soft X-ray ptychography experiments. Part of this work was performed at the Surface/Interface:Microscopy (SIM) and at the Material Science (MS) beamlines of the Swiss Light Source, Paul Scherrer Institut, Villigen, Switzerland. M. La. gratefully acknowledges funding from the European Union's Horizon 2020 research and innovation program under the 
Marie Skłodowska-Curie grant agreement No. 701647.

\section{References}

[1] B. Schmitt et al., Nucl. Instr. Meth. Phys. Res. A 518, 436 (2004). DOI: 10.1016/j.nima.2003.11.051

[2] A. Bergamaschi et al., Synchrotron Radiation News 27, 3 (2014). DOI: 10.1080/089940886.2014.930790

[3] R. Dinapoli et al., Journal of Instrumentation 9, C05015 (2014). DOI:10.1088/17480221/9/05/C05015

[4] M. Ramilli et al., Journal of Instrumentation 12, C01071 (2017). DOI: 10.1088/17480221/12/01/C010711071

[5] B. Henrich et al., Nucl. Instr. Meth. Phys. Res. A 633, S11 (2011). DOI: 10.1016/j.nima.2010.06.107.

[6] A. Bergamaschi et al., Journal of Instrumentation 10, C01033 (2015). DOI: 10.1088/17480221/10/01/C01033

[7] A. P. Hitchcock et al., Journal of Electron Spectroscopy and Related Phenomena 200, 49-63 (2015). DOI: 10.1016/j.elspec.2015.05.013

[8] D. Liebschner et al., Acta Crystallographica D72, 728-741 (2016). DOI: 10.1107/S2059798316005349

[9] G. Ghiringhelli et al., Science 337, 821-825 (2012). DOI: 10.1126/science.1223532

[10] J. Fink et al., Rep. Prog. Phys. 76, 056502 (2013). DOI: 10.1088/00344885/76/5/056502

[11] J. Becker et al., Nucl. Instr. Meth. Phys. Res. A 694, 82-90 (2012). DOI: 10.1016/j.nima.2012.08.008

[12] O. Sendetskyi et al., Physical Review B 93 (22), 224413 (2016). DOI: 10.1103/PhysrevB.93.224413

[13] U. Staub, et al., Journal of Synchrotron Radiation 15, 469-476 (2008). DOI: 10.1107/ S0909049508019614

[14] F. Pfeiffer, Nature Photonics 12, 9-17 (2018). DOI: 10.1038/s41566-017-0072-5

[15] C. Dullin et al., Journal of Synchrotron Radiation 25, 565 (2018). DOI: 1010.1107/S160057751701668X

[16] S. Cartier, M. Kagias et al., Journal of Synchrotron Radiation 23, 1462 (2016). DOI: 10.1107/S1600577516014788

[17] P. R. Willmott et al., Journal of Synchrotron Radiation 20, 667 (2013). DOI: 10.1107/S0909049513018475

[18] T. Huthwelker et al., manuscript in preparation. 